\title{
Limiting Analytical Solutions for Complex Saturated and Unsaturated Transport Problems
}

\author{
DANIEL M. TARTAKOVSKY \\ (Institute of Mathematics and Mechanics, Kazan University, Kazan, Russia) \\ Department of Hydrology and Water Resources, University of Arizona, Tucson, Arizona, USA
}

Received: 2 November 1993; in final form: 31 August 1994)

\begin{abstract}
Non-linear diffusion and velocity-dependent dispersion problems are under consideration. The necessary and sufficient conditions allowing the comparison of solutions to the two dimensional convection-dispersion equations with different coefficients are obtained. These conditions provide a framework within which solutions to the complex non-linear problems mentioned above can be estimated by solutions to the problems possessing analytical solvability.
\end{abstract}

Key words: hydrodynamic dispersion, unsaturated flow, nonlinear parabolic equation.

\section{Nomenclature}

$c(x, y, t)$

$C(h)=\mathrm{d} \Theta / \mathrm{d} h$

D, $D_{i j}$

$D_{L}$

$D_{m}$

$D_{T}$

G

$G_{z}, G_{w}$

$h$

$I_{n}$

$k$

$K(h)$

$L$

$m$

$\mathbf{n}(\mathbf{x}, t)$

$t$ concentration of solute in solution, $M L^{-3}$.

moisture capacity function.

hydrodynamic dispersion coefficient, a second order tensor, $L^{2} T^{-1}$.

longitudinal hydrodynamic dispersion coefficient, $L^{2} T^{-1}$.

molecular diffusion coefficient, $L^{2} T^{-1}$.

transverse hydrodynamic coefficient, $L^{2} T^{-1}$.

flow domain for the unsaturated flow problem.

flow domain and complex potential domain, respectively, for the hydrodynamic dispersion problem.

piezometric head, $L$.

given mass flux normal to the boundary, $M L T^{-1}$.

hydraulic conductivity, $L T^{-1}$.

unsaturated hydraulic conductivity, $L T^{-1}$.

continuously differentiable function with respect to all arguments

porosity.

outer normal vector to the boundary.

time, $T$. 


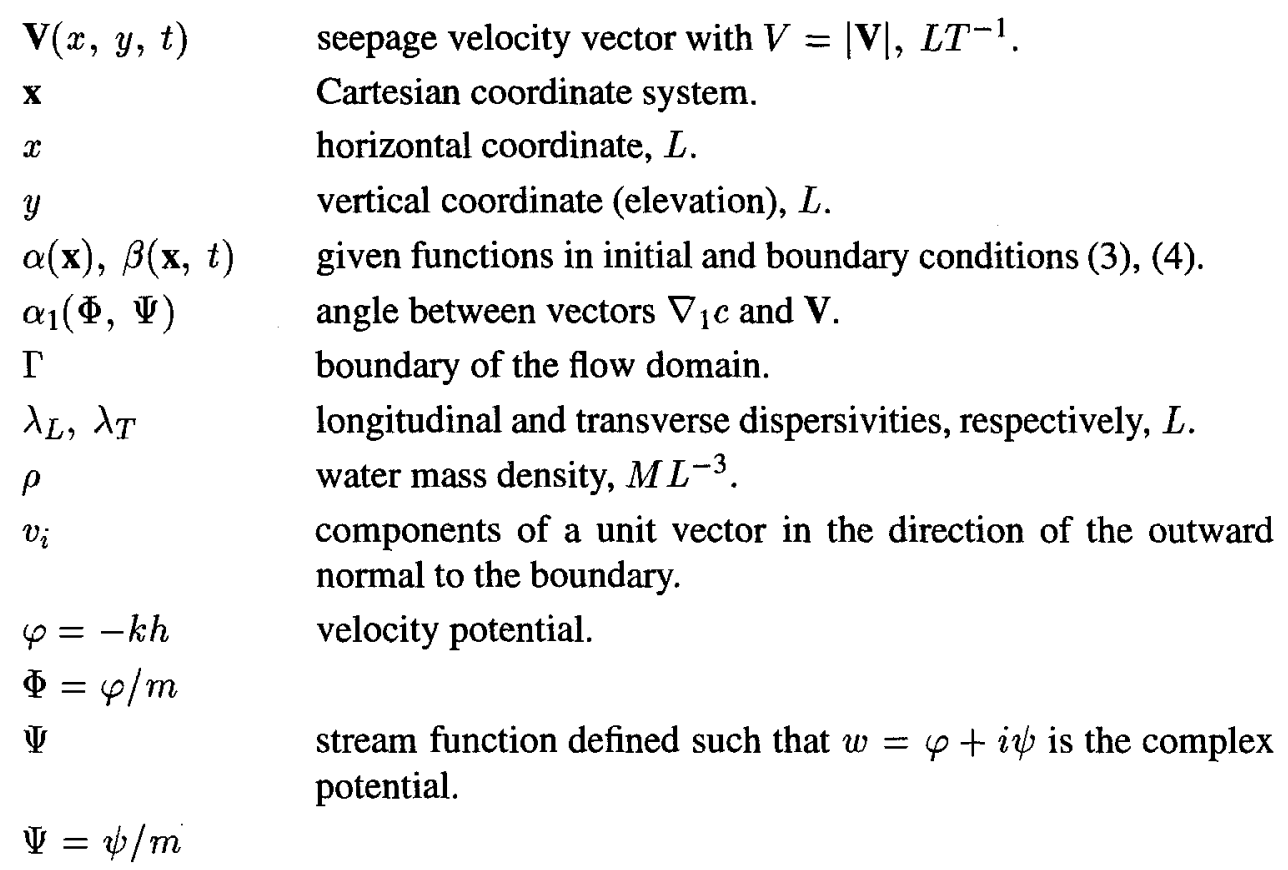

\section{Introduction}

The increase of incidents of groundwater pollution in recent years has accelerated the development of management models in the field of groundwater quality control. Although sophisticated numerical models for contaminant transport are widely used, it is obvious that application of analytical methods in solving mass and moisture transport problems offers some distinct advantages. They provide general physical insight into the transport process. Additionally, they construct a powerful tool for the validation and improvement of numerical schemes. They also form the basis for rational approximations and simplifications in terms of readily measured physical properties. Finally, they are useful for testing various inverse techniques used in the estimation of mass transport properties.

While a number of analytical solutions to the two-dimensional (2-D) convectivedispersion transport equation with constant coefficients (the seepage velocity and the dispersion coefficients) exist (see, for example, Ogata, 1976; Cleary et al., 1978; Latinopoulos et al., 1988), there is no solution available which permits a variable velocity and thus velocity-dependent dispersion coefficients.

Similar difficulties arise in solving moisture transport problems. Analytical solutions to the 1-D non-linear diffusion equation governing infiltration phenomenon have been obtained either for constant coefficients (soil water diffusivity and unsaturated hydraulic conductivity) (Horton's equation) or for coefficients which permit transformation of Richard's equation to the linear form (Gardner et al., 1958; Philip, 1974; Broadbridge et al., 1988). 
While a search for analytical solutions to problems mentioned above is continuing, non-integrability of transient "essentially" (i.e. non-reducible to 1-D equations) 2-D non-linear convection-dispersion equations has been shown (Broadbridge, 1986) and integrable forms of 1-D equation ("the Broadbridge class" of dependencies of diffusivity and conductivity on moisture content) have been given (Broadbridge, 1988).

To obtain analytical approximations for a 2-D velocity dependent dispersion equation, the methods of velocity averaging are used. These methods are based on the assumption that it is possible to average velocity over the whole seepage domain (Freeze et al., 1979, p. 395) or along flow streamlines (Dillon, 1989). In doing so, initial and boundary conditions undergo similar changes or are chosen arbitrarily to permit analytical solutions. The main disadvantage of such a procedure is, from our point of view, the unpredictable behavior of the solution to hydrodynamic dispersion equation with its coefficients being averaged. In particular, it is unclear how concentration at an arbitrary point of seepage domain and total contaminant flux are changed by averaging procedures. Therefore, for the creation of management models, estimations of the limiting values of contaminant concentration or moisture content generally turn out to be more adequate (Freeze et al., 1990). These estimations are useful for tackling such questions as whether the given quantity of contaminant can exceed the allowable concentration in water, or whether the seeping water discharge is sufficient to flood a site. The affirmative answers to such questions require more detailed investigations, while the negative ones allow us to be satisfied by estimating solutions and to avoid sophisticated numerical models.

In this paper, to obtain such estimations, we suggest using the estimating boundary-value problems which possess analytical solutions. To construct these estimating problems, we will use constant coefficients for 2-D non-linear dispersion problems and coefficients from the Broadbridge class for 1-D problems. Despite the fact that the estimations thus obtained are, in some cases, not precise, they may be considered the best ones, as has been noted above.

It is worthwhile to emphasize here that integrability of a differential equation itself does not imply solvability of the corresponding boundary-value problem. Initial and boundary conditions together with a domain's geometry are also of the utmost importance. Throughout this paper we will assume diffusion domains to permit analytical solution and will select appropriate initial and boundary conditions for the estimating problems.

Application of the comparison method of solutions to boundary-value problems for parabolic equations of the form (Galactionov et al., 1979)

$$
\partial c / \partial t=L(\mathbf{x}, c,|\nabla c|, \Delta c)
$$

(where $\mathbf{x}=x, y ; \Delta c=\nabla^{2} c$; and $L(\mathbf{x}, p, q, r)$ is a continuously differentiable function with respect to all arguments such that $\partial L / \partial r>0$, i.e. (1) is parabolic) gives necessary and sufficient conditions wherein similar estimations occur. 
As Richard's equation can be readily represented in the form (1), we will use this equation to demonstrate the statement of the theorem. The theorem will be further extended to cover a more general class of equations of the form $\partial c / \partial t=$ $L(\mathbf{x}, c, \nabla c, \Delta c)$ which includes the convective-dispersion equation.

\section{Estimations of Solution to the Unsaturated Flow Problem}

Isothermal flow of water in unsaturated soils is governed by a nonlinear diffusion equation. In terms of the piezometric head $h$ defined as $y+p / \rho g$ (where $y$ is the elevation; $p$ the pressure in the water with respect to atmospheric pressure, $\rho$ the water mass density, $g$ the gravitational acceleration) this equation for homogeneous isotropic soils takes the form

$$
C \frac{\partial h}{\partial t}=\nabla(K \nabla h)
$$

where $K(h)$ and $C=\mathrm{d} \Theta / \mathrm{d} h$ are the hydraulic conductivity of the soil and the moisture capacity, respectively; $\Theta$ is the volumetric water content.

Initial and boundary conditions of (2) are assumed to be

$$
\begin{aligned}
& h(\mathbf{x}, 0)=\alpha(\mathbf{x}), \quad \mathbf{x}(x, y, z) \in G \\
& h(\mathbf{x}, t)=\beta(\mathbf{x}, t), \quad \mathbf{x} \in \Gamma,
\end{aligned}
$$

where $\alpha$ and $\beta$ are the experimentally determined functions.

In conjunction with the problem $P,(2)-(4)$, let us consider the problem $P^{*}$

$$
\begin{aligned}
& C^{*} \frac{\partial h^{*}}{\partial t}=\nabla\left(K^{*} \nabla h^{*}\right) \\
& h^{*}(\mathbf{x}, 0)=\alpha^{*}(\mathbf{x}), \quad \mathbf{x}(x, y, z) \in G \\
& h^{*}(\mathbf{x}, t)=\beta^{*}(\mathbf{x}, t), \quad \mathbf{x} \in \Gamma .
\end{aligned}
$$

Equations (2) and (5) can be easily represented in the form (1) as

$$
\frac{\partial h}{\partial t}=\frac{K}{C} \Delta h+\frac{K^{\prime}(h)}{C}|\nabla h|^{2}, \quad K^{\prime}(h)=\frac{\partial K}{\partial h} .
$$

It follows from the comparison theorem (Appendix A) that the inequality

$$
h(\mathbf{x}, t) \leqslant h *(\mathbf{x}, t), \quad \mathbf{x} \in G
$$

takes place subject to the following conditions (Galactionov et al., 1979):

$$
\frac{K^{*}}{C^{*}} \leqslant \frac{K}{C}, \quad \mathbf{x} \in G
$$




$$
\begin{aligned}
& \frac{\left(K^{*} / K\right)^{\prime}}{C^{*} C} \geqslant 0, \quad \mathbf{x} \in G \\
& \alpha^{*}(\mathbf{x}) \geqslant \alpha(\mathbf{x}), \quad \mathbf{x} \in G \\
& \beta^{*}(\mathbf{x}, t) \geqslant \beta(\mathbf{x}, t), \quad \mathbf{x} \in \Gamma .
\end{aligned}
$$

Thus, in the 1-D case, solution to the vertical infiltration problem $P$ can be estimated by solution to the corresponding problem $P^{*}$. Additionally, if functions $K^{*}$ and $C^{*}$ belong to the Broadbridge class, and the functions $\alpha^{*}$ and $\beta^{*}$ are chosen so that they provide analytical solution, this estimation is analytical.

\section{Estimations of Solution to the Hydrodynamic Dispersion Problem}

Consider a homogeneous isotropic plane aquifer under steady-state saturated flow with seepage velocity being variable in space. Suppose the velocity field $\mathbf{V}(u(x, y), v(x, y))$ was determined by solving a boundary value problem for the Darcy and conservation equations

$$
\mathbf{V}=-k \nabla h=\nabla \varphi \quad \nabla \cdot \mathbf{V}=0
$$

where $k$ is the hydraulic conductivity; $h$ the piezometric head defined as above for the vertical plane and as $p / \rho g$ for the horizontal plane; $\varphi$ the velocity potential.

Assume $\mathbf{V}(x, y)$ to be a limited function $(V=|\mathbf{V}|<\infty)$ on the boundary $\Gamma_{2}$ of the seepage domain $G_{z}$. As the seepage velocity vector $\mathbf{V}$ is a potential vector, that assumption implies that $V<\infty$ over the whole domain $G_{z}$.

Under these conditions, transport of a conservative solute can be described by the 2-D advective-dispersive differential equation in Cartesian coordinates system (Bear, 1972, p. 617)

$$
\frac{\partial c}{\partial t}=\nabla(\mathbf{D} \nabla c)-\mathbf{V} \nabla c
$$

where $c$ is the concentration of solute in solution, equal to $c(x, y, t) ; t$ time; $\nabla c=(\partial c / \partial x, \partial c / \partial y) ; x, y$ are Cartesian coordinates; and $\mathbf{D}$ the hydrodynamic dispersion coefficient, a second-order tensor.

The initial concentration $c_{0}$ is assumed to be an arbitrary function

$$
c(x, y, 0)=c_{0}(x, y), \quad(x, y) \in G_{z} .
$$

The conditions along the boundaries of a flow domain $G_{z}$ throughout which hydrodynamic dispersion occurs are based on the requirement that for any point of the boundary surface, the solute's mass flux normal to the boundary must be equal on both sides of a stationary boundary (Bear, 1972, p. 623)

$$
m v_{i}\left(-D_{i j} \frac{\partial c}{\partial x_{j}}+V_{i} c\right)=I_{n}
$$


where $I_{n}$ is a given mass flux (mass per unit length of the boundary) normal to a boundary $\Gamma_{x} ; m$ is the porosity; and $v_{i}$ denote components of a unit vector in the direction of the outward normal to the boundary.

In the orthogonal curvilinear coordinate system $\Phi, \Psi$, the problem $P,(15)-(17)$, is written as (Bear, 1972, p. 620)

$$
\begin{aligned}
& \frac{\partial c}{\partial t}=V^{2}(\Phi, \Psi)\left[\frac{\partial}{\partial \Phi}\left(D_{L} \frac{\partial c}{\partial \Phi}\right)+\frac{\partial}{\partial \Psi}\left(D_{T} \frac{\partial c}{\partial \Psi}\right)-\frac{\partial c}{\partial \Phi}\right] \\
& c(\Phi, \Psi, 0)=c_{0}(\Phi, \Psi), \quad(\varphi, \psi) \in G_{w} \\
& V\left(-v_{\Phi} D_{L} \frac{\partial c}{\partial \Phi}-v_{\Psi} D_{T} \frac{\partial c}{\partial \Psi}+c\right)=I_{n}, \quad(\varphi, \psi) \in G_{w}
\end{aligned}
$$

where $\Phi=\varphi / m ; \Psi=\psi / m ; \psi$ is a stream function; $G_{w}$ the complex potential domain with boundary $\Gamma_{w} ; w=\varphi+i \psi$ the complex potential plain; $v_{\Phi}$ and $v_{\Psi}$ the components of a unit vector in the direction of the outward normal to the boundary $\Gamma_{w}$.

The longitudinal and transverse hydrodynamic dispersion coefficients, $D_{L}$ and $D_{T}$, are further expressed as

$$
D_{L}=D_{m}+\lambda_{L} V, \quad D_{T}=D_{m}+\lambda_{T} V
$$

where $\lambda_{L}$ and $\lambda_{T}$ are the longitudinal and transverse dispersivities; $D_{m}$ the molecular diffusion coefficient.

Along the line of Section 2 let us introduce the estimating boundary value problem $P^{*}$ defined as follows

$$
\begin{aligned}
& \frac{\partial c^{*}}{\partial t}=V^{* 2}\left[D_{L}^{*} \frac{\partial^{2} c^{*}}{\partial \Phi^{2}}+D_{T}^{*} \frac{\partial^{2} c^{*}}{\partial \Psi^{2}}-\frac{\partial c^{*}}{\partial \Phi}\right] \\
& V^{*}\left(-v_{\Phi} D_{L}^{*} \frac{\partial c^{*}}{\partial \Phi}-v_{\Psi} D_{T}^{*} \frac{\partial c^{*}}{\partial \Psi}+c^{*}\right)=I_{n}^{*} \quad(\varphi, \psi) \in G_{w} \\
& c^{*}(\Phi, \Psi, 0)=c_{0}^{*}(\Phi, \Psi), \quad(\varphi, \psi) \in G_{w} \\
& D_{L}^{*}=D_{m}+\lambda_{L} V^{*}, \quad D_{T}^{*}=D_{m}+\lambda_{T} V^{*}
\end{aligned}
$$

where $V^{*}$ is a constant over the whole domain (or along any streamline $\psi=$ const) coefficient defined as

$$
\begin{aligned}
& V^{*}=\left|\boldsymbol{V}^{*}\right|=\max |V(\varphi, \psi)|\left(V_{\psi}^{*}=\max \mid V(\varphi, \psi=\text { const }) \mid\right) \\
& \boldsymbol{V}^{*} \| \boldsymbol{V}, \quad(\varphi, \psi) \in G_{w}
\end{aligned}
$$


and, thus, $D_{L}^{*}$ and $D_{T}^{*}$ are constants.

Then, taking into account that $D_{L}$ and $D_{T}$ are positive functions, the inequality

$$
c(\Phi, \Psi, t) \leqslant c *(\Phi, \Psi, t), \quad(\varphi, \psi) \in G_{w}
$$

is valid subject to the following conditions (see Appendix B)

$$
\begin{aligned}
& c_{0}^{*}(\Phi, \Psi) \geqslant c_{0}(\Phi, \Psi), \quad(\varphi, \psi) \in G_{w} ; \quad I_{n}^{*} \leqslant I_{n}, \quad(\varphi, \psi) \in \Gamma_{w} \\
& \begin{aligned}
& \frac{\partial c^{*}(\Phi, \Psi, t)}{\partial t} \geqslant 0, \quad(\varphi, \psi) \in G_{w} \\
& \frac{\cos \alpha_{1}^{*}(\Phi, \Psi)}{V^{* 2} \sqrt{\lambda_{L}}}+ {\left[\left(\lambda_{L} \frac{\partial V}{\partial \Phi}-1\right) \cos \alpha_{1}(\Phi, \Psi)\right.} \\
&\left.+\sqrt{\lambda_{L} \lambda_{T}} \frac{\partial V}{\partial \Psi} \sin \alpha_{1}(\Phi, \Psi)\right] \frac{1}{V^{2}(\Phi, \Psi)} \leqslant 0
\end{aligned}
\end{aligned}
$$

where $\alpha_{1}^{*}$ and $\alpha_{1}$ are angles between vectors $\nabla_{1} c^{*}$ and $\mathbf{V}^{*}, \nabla_{1} c$ and $\mathbf{V}$ respectively; $\nabla_{1}=\left(\lambda_{L}^{1 / 2} \partial / \partial \Phi, \lambda_{T}^{1 / 2} \partial / \partial \Psi\right)$.

By these means, the problem $P^{*}$ solution can serve as an estimation of the problem $P$ solution subject to the conditions (28)-(30).

Inequality (29), being the definition of a "critical" solution to a boundary value problem, is equivalent to the conditions (Galactionov et al., 1979)

$$
\begin{aligned}
& D_{L}^{*} \frac{\partial^{2} c_{0}^{*}}{\partial \Phi^{2}}+D_{T}^{*} \frac{\partial^{2} c_{0}^{*}}{\partial \Psi^{2}}-\frac{\partial c_{0}^{*}}{\partial \Phi} \geqslant 0, \quad(\varphi, \psi) \in G_{w} ; \\
& \frac{\partial I_{n}^{*}}{\partial t} \geqslant 0, \quad(\varphi, \psi) \in \Gamma_{w} .
\end{aligned}
$$

Conditions (28) compare both known initial concentration distribution over $G_{w}$ and known concentration flux through its boundary $\Gamma_{w}$ with ones providing analytical solution. Thus, in conjunction with conditions $\left(29^{\prime}\right)$, they outline the class of initial and boundary conditions to the problem $P^{*}$.

Condition (30) states an obvious fact that the concentration variation degree depends on the angle between the flow velocity vector and the direction of concentration gradient in a flow domain. That dependence, however, is not trivial and testing condition (30) is of great difficulty since prior knowledge of solutions to both $P$ and $P^{*}$ problems is needed. Nevertheless, for a vast class of problems it is enough to know only the variation interval of the angle $\alpha_{1}$, which can be obtained from field tests without solving the problem. 


\subsection{MASS TRANSPORT WITH $D_{T}=0$}

In the case of the large distance traveled, the following simplification can be gained by assuming that transverse hydrodynamic dispersivity is neglected $\left(D_{T}=0\right)$. Then (5) is reduced to

$$
\frac{\partial c}{\partial t}=V^{2}(\Phi, \Psi)\left[\frac{\partial}{\partial \Phi}\left(D_{L} \frac{\partial x}{\partial \Phi}\right)-\frac{\partial c}{\partial \Phi}\right] .
$$

If it is known (from field experiment) that $\partial c / \partial \Phi \leqslant 0(\partial c / \partial \Phi \geqslant 0)$ along any streamline $\Psi=$ constant for the time under consideration, equation (17) can be represented in the form (1):

$$
\frac{\partial c}{\partial t}=V^{2}(\Phi, \Psi)\left[D_{L} \frac{\partial^{2} c}{\partial \Phi^{2}}-(+)\left(\lambda_{L} \frac{\partial V}{\partial \Phi}-1\right)\left|\frac{\partial c}{\partial \Phi}\right|\right] .
$$

Under these assumptions solution to the velocity dependent dispersion problem (31), (19), (20) can be estimated by solution to the equation

$$
\frac{\partial c^{*}}{\partial t}=V^{* 2}\left[D_{L}^{*} \frac{\partial^{2} c^{*}}{\partial \Phi^{2}}+(-) \frac{\partial c^{*}}{\partial \Phi}\right]
$$

subject to initial and boundary conditions (23), (24).

Here $V^{*}$ should be selected to satisfy the following inequalities

$$
\begin{aligned}
& V^{*} \geqslant V(\Phi, \Psi), \quad(\varphi, \psi) \in G_{w} \\
& -(+) \frac{D_{L}}{D_{L}^{*}}-(+)\left(\lambda_{L} \frac{\partial V}{\partial \Phi}-1\right) \leqslant 0, \quad(\varphi, \psi) \in G_{w} .
\end{aligned}
$$

For mass transport with negligible transverse dispersivity all of the necessary and sufficient conditions (28), (29), (30') of existence of the majorizing estimation (27) can be verified a-priory. As an example, in the case of the steady flow, the known analytical solution to the estimating problem (Polubarinova-Kochina, 1962, p. 356) can be used as such an estimation.

In a similar manner, it can be shown that the diffusion equation $(V=0, D=$ $D_{m}$ ) solution subject to corresponding initial and boundary conditions is the minorizing estimation of the problem $P$.

\section{Conclusion}

In this paper, the necessary and sufficient conditions of existence of the estimations to the linear velocity dependent dispersion problem and the non-linear diffusion problem have been obtained. In doing so, solutions of the estimating equations are used. The Broadbridge class of hydraulic conductivity and moisture capacity 
functions is wide enough to cover many practical problems. Choice of initial and boundary conditions satisfying inequalities (12), (13) allows analytical estimation of infiltration parameters (time to ponding, depth of wetting front, etc.) for arbitrary rainfall event and soil water parameters from the Broadbridge class. In the case of 2-D dispersion, accuracy of estimation (27) is obviously a function of the seepage velocity variability over the domain. The greater the velocity change along a streamline, the more approximate is the estimation obtained, but, let us stress it again, it is best one.

Some multi-dimensional transport problems with variable coefficients (transport by radial flow, for example) allow reduction to 1-D convection-dispersion equation (Yates, 1988) and, thus, analytical solvability. These solutions can be used as estimations to the corresponding classes of transport problems, and necessary and sufficient conditions, wherein these estimations occur, can be found using procedure outlined in this paper.

\section{Appendix A}

\section{Statement of the direct comparison theorem}

As material used as a reference in this paper is hardly accessible for non-Russian speaking readers, we reproduce here the relevant theorems of the direct comparison method (as they are stated by Samarskii et al., 1989, p. 289).

Let $\Omega$ be an arbitrary domain (not necessary finite) in $\mathbf{R}^{N}$ with a smooth boundary $\partial \Omega$. For non-linear parabolic equation

$$
\partial u / \partial t=L(u,|\nabla u|, \Delta u)
$$

consider the boundary value problem with initial and boundary conditions

$$
\begin{aligned}
& u(\mathbf{x}, 0)=u_{0}(\mathbf{x}) \geqslant 0, \quad \mathbf{x} \in \Omega \\
& u(\mathbf{x}, t)=u_{1}(\mathbf{x}, t) \geqslant 0, \quad \mathbf{x} \in \partial \Omega .
\end{aligned}
$$

It is assumed that $u_{0}(\mathbf{x}) \rightarrow 0, u_{1}(\mathbf{x}, t) \rightarrow 0$ with $|\mathbf{x}| \rightarrow \infty$ and $u(\mathbf{x}, t) \rightarrow 0$ with $|\mathbf{x}| \rightarrow \infty$ for any $t$. Function $L(p, q, r)$ in (A1) is defined and once continuously differentiable function with respect to all arguments. Moreover, $\partial L / \partial r>0$ in $\mathbf{R}_{+} \times \mathbf{R}_{+} \times \mathbf{R}$ that means parabolicity of equation (A1).

It is also assumed that there is a real function $r=l(p, q, Y)$ such that

$$
L(p, q, l(p, q, Y))=Y, \quad(p, q, Y) \in \mathbf{R}_{+} \times \mathbf{R}_{+} \times \mathbf{R}
$$

and $l_{0}(p, q)=l(p, q, 0)$.

DEFINITION. Problem (A1)-(A3) and its solution $u(\mathbf{x}, t)$ is called the critical ones if for any $t$ and $\mathbf{x} \in \Omega$ the inequalities

$$
u(\mathbf{x}, t) \geqslant 0, \quad \partial u / \partial t \geqslant 0
$$


hold true.

THEOREM 1. For the problem (A1)-(A3) to be critical, the following conditions are necessary and sufficient:

$$
\begin{aligned}
& L\left(u_{0},\left|\nabla u_{0}\right|, \Delta u_{0}\right) \geqslant 0, \quad \mathbf{x} \in \Omega \\
& \partial u_{1}(\mathbf{x}, t) / \partial t \geqslant 0, \quad \mathbf{x} \in \partial \Omega .
\end{aligned}
$$

Consider boundary problems for two different parabolic equations $(i=1,2)$ :

$$
\begin{aligned}
& \partial u^{(i)} / \partial t=L^{(i)}\left(u^{(i)},\left|\nabla u^{(i)}\right|, \Delta u^{(i)}\right) \\
& u^{(i)}(\mathbf{x}, t)=u_{0}^{(i)}(\mathbf{x}) \geqslant 0, \quad \mathbf{x} \in \Omega \\
& u^{(i)}(\mathbf{x}, t)=u_{1}^{(i)}(\mathbf{x}, t), \quad \mathbf{x} \in \partial \Omega .
\end{aligned}
$$

THEOREM 2. Let $u^{(2)} \geqslant u^{(1)}$ on the boundary, i.e.

$$
u_{0}^{(2)}(\mathbf{x}) \geqslant u_{0}^{(1)}(\mathbf{x}), \quad \mathbf{x} \in \Omega, \quad u_{1}^{(2)}(\mathbf{x}, t) \geqslant u_{1}^{(1)}(\mathbf{x}, t), \quad \mathbf{x} \in \partial \Omega .
$$

Moreover, let the solution to the problem (A8)-(A10) for $i=2$ be critical and for all $(p, q, r) \in \mathbf{R}_{+} \times \mathbf{R}_{+} \times \mathbf{R}$ the following conditions hold true:

$$
\begin{aligned}
& \frac{\partial}{\partial r}\left[L^{(2)}(p, q, r)-L^{(1)}(p, q, r)\right] \geqslant 0 \\
& L^{(1)}\left(p, q, l_{0}^{(2)}(p, q)\right) \leqslant 0 .
\end{aligned}
$$

Then $u^{(2)} \geqslant u^{(1)}$ in the whole domain $(\mathbf{x} \in \Omega, t>0)$.

Furthermore, it is pointed out that these theorems are valid for more general boundary conditioins in the form

$$
-\partial u / \partial n+u=u_{1}(x, t), \quad \mathbf{x} \in \partial \Omega
$$

\section{Appendix B}

Derivation of the conditions (28)-(30)

Assuming the contribution of molecular diffusion to contaminant transport process to be small, in derivation of conditions (28)-(30) we put $D_{m}=0$. Then equations (18) and (22) can be written as

$$
\frac{\partial c}{\partial t}=V^{3} \lambda_{L} \frac{\partial^{2} c}{\partial \Phi^{2}}+V^{3} \lambda_{T} \frac{\partial^{2} c}{\partial \Psi^{2}}+\lambda_{L} \frac{\partial V}{\partial \Phi} \frac{\partial c}{\partial \Phi}+\lambda_{T} \frac{\partial V}{\partial \Psi} \frac{\partial c}{\partial \Psi}-V_{\Phi} \frac{\partial c}{\partial \Phi}
$$




$$
\frac{\partial c^{*}}{\partial t}=V^{* 3} \lambda_{L} \frac{\partial^{2} c^{*}}{\partial \Phi^{2}}+V^{* 3} \lambda_{T} \frac{\partial^{2} c^{*}}{\partial \Psi^{2}}-V_{\Phi}^{*} \frac{\partial c^{*}}{\partial \Phi}
$$

where $\left(a_{i}\right)$ are "physical components" of a vector a defined for the given coordinate system $\Phi-\Psi$ as $\left(a_{i}\right)=V\left(a_{i}\right)$.

It follows from (B1) and (B2) that

$$
\begin{aligned}
& \frac{\partial c}{\partial t}=V^{3} \lambda_{L} \frac{\partial^{2} c}{\partial \Phi^{2}}+V^{3} \lambda_{T} \frac{\partial^{2} c}{\partial \Psi^{2}}+|\mathbf{q}|\left|\nabla_{c}\right| \cos \beta-|\mathbf{V}||\nabla c| \cos \alpha \\
& \frac{\partial c^{*}}{\partial t}=V^{* 3} \lambda_{L} \frac{\partial^{2} c^{*}}{\partial \Phi^{2}}+V^{* 3} \lambda_{T} \frac{\partial^{2} c^{*}}{\partial \Psi^{2}}-\left|\mathbf{V}^{*}\right|\left|\nabla c^{*}\right| \cos \alpha^{*},
\end{aligned}
$$

where $\mathbf{q}=\lambda_{L}(\partial V / \partial \Phi) \mathbf{e}_{\Phi}+\lambda_{T}(\partial V / \partial \Psi) \mathbf{e}_{\Psi} ; \mathbf{V}=\left(V_{\Phi}\right) \mathbf{e}_{\Phi},\left(V_{\Phi}\right)=V ; \mathbf{e}_{\Phi}$ and $\mathbf{e}_{\Psi}$ are unit vectors directed along the normals to the coordinate curves $\varphi=$ const and $\psi=$ const, respectively; $\alpha, \beta, \alpha^{*}$ are angles between vectors $\mathbf{V}$ and $\nabla c, \mathbf{q}$ and $\nabla c, \mathbf{V}^{*}$ and $\nabla c^{*}$, respectively.

To bring equations (B3) and (B4) to the form (1), we introduce new coordinates $\Phi_{1}=\left(\lambda_{L}\right)^{1 / 2} \Phi, \Psi_{1}=\left(\lambda_{T}\right)^{1 / 2} \Psi$. Then equations (B3) and (B4) are

$$
\begin{aligned}
& \frac{\partial c}{\partial t}=V^{3} \Delta_{1} c+\left|\nabla_{1} V\right| \cos \beta_{1}\left|\nabla_{1} c\right|-\frac{V}{\sqrt{\lambda_{L}}} \cos \alpha_{1}\left|\nabla_{1} c\right| \\
& \frac{\partial c^{*}}{\partial t}=V^{* 3} \Delta_{1} c^{*}-\frac{V^{3}}{\sqrt{\lambda_{L}}} \cos \alpha_{1}^{*},
\end{aligned}
$$

where $\nabla_{1}=\left(\partial / \partial \Phi_{1}, \partial / \partial \Psi_{1}\right) ; \alpha_{1}, \beta_{1}, \alpha_{1}^{*}$ are angles corresponding to angles $\alpha_{1}, \beta_{1}, \alpha_{1}^{*}$ in the new coordinate system. Then, according to the comparison theorem, inequality

$$
c \leqslant c^{*}
$$

holds true subject to the following conditions

$$
\begin{aligned}
& c_{0}^{*}\left(\Phi_{1}, \Psi_{1}\right) \geqslant c_{0}\left(\Phi_{1}, \Psi_{1}\right), \quad(\varphi, \psi) \in G_{w} \\
& I_{n}^{*}\left(\Phi_{1}, \Psi_{1}\right) \leqslant I_{n}\left(\Phi_{1}, \Psi_{1}\right), \quad(\varphi, \psi) \in \Gamma_{w} \\
& \frac{\partial c^{*}\left(\Phi_{1}, \Psi_{1}, t\right)}{\partial t} \geqslant 0, \quad(\varphi, \psi) \in G_{w} \\
& \frac{\cos \alpha_{1}^{*}}{V^{* 2} \sqrt{\lambda_{L}}}+\frac{\left|\nabla_{1} V\right| \cos \beta_{1}-V \cos \alpha_{1} / \sqrt{\lambda_{L}}}{V^{3}} \leqslant 0, \quad(\varphi, \psi) \in G_{w},
\end{aligned}
$$




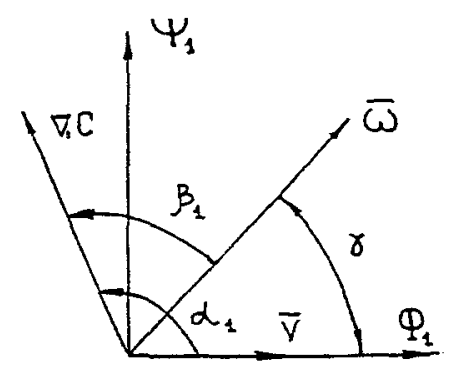

Fig. 1. To derivation of the formula (B12). The isotropic $\Phi_{1}-\Psi_{1}$ coordinate system is defined by heterogeneous transformation of the equipotential-streamline coordinate system as $\Phi_{1}=\left(\lambda_{1}\right)^{1 / 2} \Phi, \Psi_{1}=\left(\lambda_{T}\right)^{1 / 2} \Psi$. Vector $\omega$ is an image of the vector $q$ in the new coordinate system. $\alpha_{1}, \beta_{1}$, and $\gamma$ are the angles between vectors $\mathbf{V}$ and $\nabla_{1} c, \omega$ and $\nabla_{1} c, \mathbf{V}$ and $\omega$, respectively, in the $\Phi_{1}-\Psi_{1}$ plain.

\section{Taking into account the following relationships (see Figure 1):}

$$
\begin{aligned}
& \cos \beta_{1}=\cos \left(\alpha_{1}-\gamma\right)=\cos \alpha_{1} \cos \gamma+\sin \alpha_{1} \sin \gamma \\
& \gamma=\arccos \left(\frac{\partial V / \partial \Phi_{1}}{\left|\nabla_{1} V\right|}\right)=\arcsin \left(\frac{\partial V / \partial \Psi_{1}}{\left|\nabla_{1} V\right|}\right)
\end{aligned}
$$

inequality (B11) finally yields

$$
\frac{\cos \alpha_{1}^{*}}{V^{* 2}}+\frac{\left(\lambda_{L} \partial V / \partial \Phi_{1}-1\right) \cos \alpha_{1}+\sqrt{\lambda_{L} \lambda_{T}} \sin \alpha_{1} \partial V / \partial \Psi_{1}}{V^{2}} \leqslant 0 .
$$

\section{References}

Bear, J., 1972, Dynamic of Fluids in Porous Media, Elsevier Sci., New York.

Broadbridge, P., 1986, Nonintegrability of nonlinear diffusion-convection equations in two spatial dimensions, J. Phys. A: Math. Gen. 19, 1245-1257.

Broadbridge, P. and White, I., 1988, Constant rate rainfall infiltration: a versatile nonlinear model. 1. Analytical solution, Wat. Res. Res. 24, No. 1, 145-154,

Broadbridge, P., 1988, Integrable forms of the one-dimensional flow equation for unsaturated heterogeneous porous media, J. Math. Phys, 29, 622-627.

Cleary, R. W. and Ungs, M. J., 1978, Groundwater pollution and hydrology, mathematical models and computer programs, Rep. 78-WR-15, Water Resour. Program, Princeton Univ., Princeton, N.J.

Dillon, P. J., 1989, An analytical model of contaminant transport from diffuse sources in saturated porous media, Wat. Res. Res. 25, No. 6, 1208-1218.

Freeze, R. A. and Cheery, J. A., 1979, Groundwater, Prentice-Hall, Inc., New Jersey.

Freeze, R. A., Massmann, J., Smith, L., Sperling, T. and James, B., 1990, Hydrogeological decision analysis: 1. a framework, Ground Water 28, No. 5, 738-766.

Galactionov, V. A., Kurdiumov, S. P., Mikhailov, A. P. and Samarskii, A. A., 1979, On the comparison of solutions to parabolic equations, Doklady Akademii Nauk USSR 248, No. 3, 586-594 (in Russian).

Gardner, W. R. and Mayhugh, M. S., 1958, Solutions and tests of the diffusion equation for the movements of water in soil, Proc. Soil Sci. Soc. Am. 22, No. 3, 197-201. 
Latinopoulos, P., Tolikas, D. and Mylopoulos, Y., 1988, Analytical solutions for two-dimensional chemical transport in aquifers, Journal of Hydrology 98, 11-19.

Ogata, A., 1976, Two-dimensional steady-state dispersion in a saturated porous medium, Jour. Research U.S. Geol. Survey 4, No. 3, 277-284.

Philip, J. R., 1974, Recent progress in the solution of nonlinear diffusion equations, Soil Sci. 117, 257-264.

Polubarinova-Kochina, P. Ja., 1962, Theory of Ground Water Movement, Princeton Univ. Press, Princeton, N.Y.

Samarskii, A. A., Galactionov, V. A., Kurdiumov, S. P. and Mikhailov, A. P., 1987, Regimy s Obostrenijami $\vee$ Zadatchach dlja Kvazilineinych Parabolitcheskich Uravnenii, Nauka, Moscow (in Russian).

Yates, S. R., 1988, Three-dimensional radial dispersion in a variable velocity flow field, Wat. Res. Res. 24, No. 7, 1083-1090. 